\title{
Correction to: The impact of caregiver's role preference on decisional conflicts and psychiatric distresses in decision making to help caregiver's disclosure of terminal disease status
}

\author{
Shin Hye Yoo ${ }^{1}$. Young Ho Yun ${ }^{2}\left({ }^{0} \cdot\right.$ Kyoung-Nam Kim ${ }^{3}$. Jung Lim Lee ${ }^{4}$. Jeanno Park ${ }^{5}$ Youn Seon Choi ${ }^{6}$. \\ Yeun Keun $\mathrm{Lim}^{7}$. Samyong Kim ${ }^{8} \cdot$ Hyun Sik Jeong ${ }^{9}$. Jung Hun Kang ${ }^{10} \cdot$ Ho-Suk Oh ${ }^{11}$. Ji Chan Park ${ }^{12}$. \\ Si-Young $\mathrm{Kim}^{13} \cdot$ Hong Suk Song $^{14} \cdot \mathrm{Keun}$ Seok Lee ${ }^{15}$. Dae Seog Heo ${ }^{1}$. Young Seon Hong ${ }^{16}$
}

Published online: 21 February 2019

๑) Springer Nature Switzerland AG 2019

\section{Correction to: \\ Quality of Life Research (2018) 27:1571-1581 \\ https://doi.org/10.1007/s11136-018-1814-7}

In the original publication of the article, the incorrect grant number $\mathrm{HC} 13 \mathrm{C} 1391$ was published in the acknowledgement section. The correct grant number is HC15C1391.

Publisher's Note Springer Nature remains neutral with regard to jurisdictional claims in published maps and institutional affiliations.

The original article can be found online at https://doi.org/10.1007/ s11136-018-1814-7.

Young Ho Yun

lawyun@snu.ac.kr

1 Department of Internal Medicine, Seoul National University College of Medicine, Seoul, Republic of Korea

2 Department of Biomedical Science, Seoul National University College of Medicine, 103 Daehak-ro, Jongno-gu, Seoul 110-799, Republic of Korea

3 Institute of Public Health and Medical Service, Seoul National University Hospital, Seoul, Republic of Korea

4 Department of Hemato-Oncology, Daegu Fatima Hospital, Daegu, Republic of Korea

5 Palliative Care and Hospice Center, Bobath Memorial Hospital, Bundang, Seongnam, Republic of Korea

6 Department of Family Medicine, Korea University Guro Hospital, Seoul, Republic of Korea

7 Department of Internal Medicine, Kwangju Christian Hospital, Kwangju, Republic of Korea

8 Division of Hemato-Oncology, Department of Internal Medicine, College of Medicine, Chungnam National University, Daejeon, Republic of Korea
9 Department of Hematology and Oncology, GSAM Hospital, Gunpo, Republic of Korea

10 Department of Internal Medicine, Postgraduate Medical School, Gyeongsang National University, Jinju, Republic of Korea

11 Department of Internal Medicine, Gangneung Asan Hospital, University of Ulsan College of Medicine, Gangneung, Republic of Korea

12 Division of Hemato-Oncology, Department of Internal Medicine, Daejeon St Mary's Hospital, The Catholic University of Korea, Daejeon, Republic of Korea

13 Departments of Medical Oncology and Hematology, Kyung Hee University Hospital, Seoul, Republic of Korea

14 Department of Internal Medicine, Keimyung University Dongsan Medical Center, Daegu, Republic of Korea

15 Center for Breast Cancer, Research Institute and Hospital, National Cancer Center, Goyang, Republic of Korea

16 Department of Internal Medicine, Seoul St Mary's Hospital, The Catholic University of Korea, Seoul, Republic of Korea 